\title{
Rückblick: 10 Jahre neuroreha
}

\author{
Susanna Freivogel
}

\author{
Seit 10 Jahren bietet die Zeitschrift „neuroreha“ einen wahren Schatz therapeutisch \\ relevanter Informationen. Sie hilft Therapeuten, diejenigen Maßnahmen in der Therapie \\ einzusetzen, die sich in wissenschaftlichen Studien als erfolgreich erwiesen haben. \\ Gründungsherausgeberin Susanna Freivogel zeigt auf, wie Zeitschriften helfen können, \\ die Therapiewelt zu verändern.
}

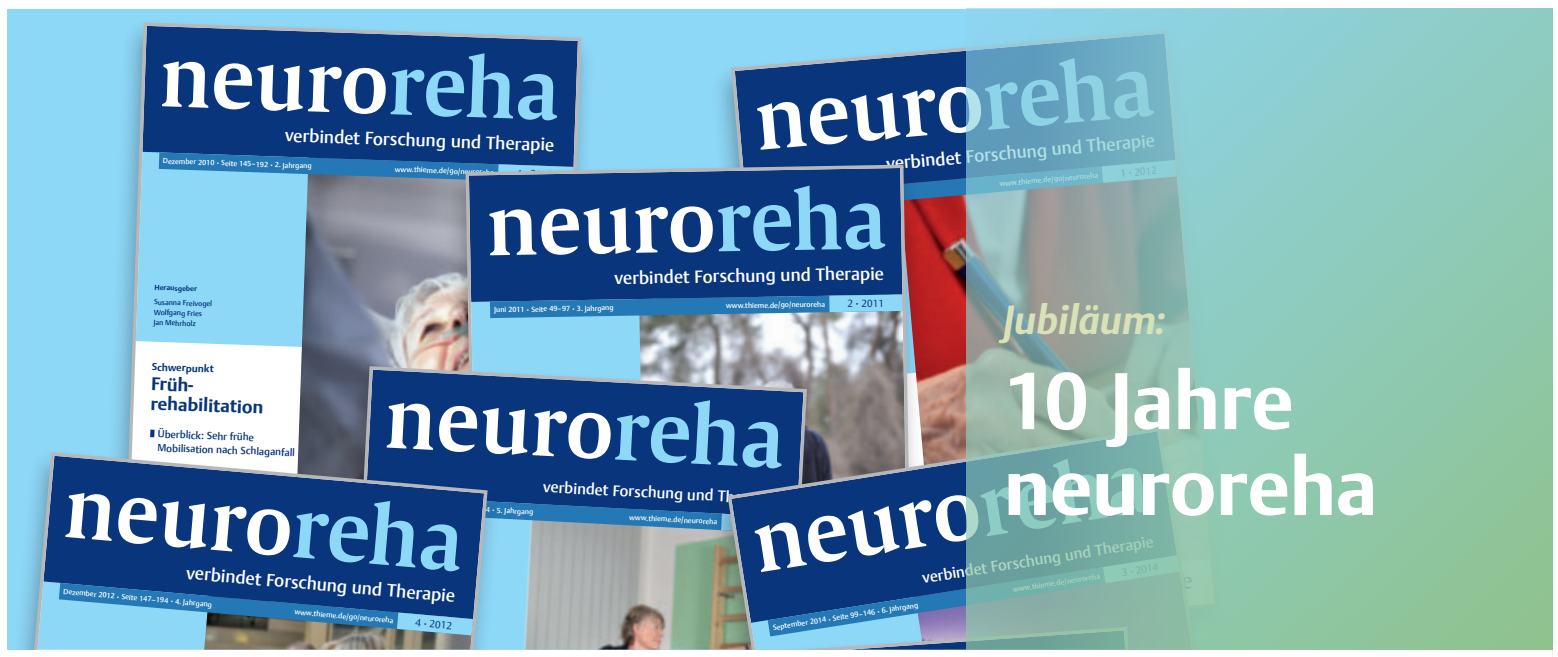

Seit 10 Jahren überbrückt die neuroreha die Lücke zwischen wissenschaftlichen Erkenntnissen und therapeutischer Umsetzung in den klinischen Alltag.

Ziel der Therapie in der neurologischen Rehabilitation ist - nach Maßgabe des Sozialgesetzbuches IX - die bestmögliche funktionelle Erholung als Voraussetzung für eine selbstbestimmte Aktivität und Teilhabe des Patienten. SGB V fordert mit §12, dass die dafür eingesetzten Therapien zweckmäßig und wirtschaftlich sein müssen. Um das zu gewährleisten, müssen in der Therapie diejenigen Maßnahmen angewandt werden, die sich in wissenschaftlichen Studien als erfolgreich erwiesen haben. Aus diesem Grunde ist eine Beschäftigung mit Studien zur Wirksamkeit des therapeutischen Vorgehens unabdingbar.

Während im englischsprachigen Raum die Diskussion therapeutischer Behandlungsansätze seit längerer Zeit stattfindet und die sogenannten neurophysiologischen Behandlungskonzepte (Bobath, Propriozeptive Neuromuskuläre Fazilitation, Vojta) wegen fehlender Evidenz einer überlegenen Wirksamkeit, unzureichender theoretischer Rahmenkonzeptionen und konzeptspezifischer Terminologie infrage gestellt werden, repräsentier(t)en sie in Deutschland immer noch den „Goldstandard“ des therapeutischen Vorgehens. Grund dafür ist zum einen die feh- lende Auseinandersetzung mit Ergebnissen wissenschaftlicher Studien und zum anderen die in Deutschland im Heilmittelkatalog definierte besondere Position von „KG-ZNS“ und der daraus resultierenden höheren Vergütungen von „neurophysiologischen Techniken (Bobath, Vojta, PNF)“ in der ambulanten Physiotherapie.

\section{neuroreha füllt die Lücke zwischen Forschung und Therapie}

Es ist dem Thieme Verlag und hier insbesondere Rosi Haarer-Becker sehr herzlich zu danken, dass sie das Spannungsfeld zwischen wissenschaftlichen Erkenntnissen und deren therapeutischer Umsetzung in den klinischen Alltag gesehen haben und bereit waren, 2008 eine Zeitschrift zu lancieren, die die Lücke zwischen Forschung und Therapie schließen wollte.

Konzipiert wurde die neuroreha mit jährlich vier themenspezifischen Heften mit jeweils drei bis vier wissenschaftlich orientierten Übersichtsartikeln und zwei praxisorien- 
tierten Beiträgen, die basierend auf dem aktuellen Wissensstand therapeutische Anwendungen im Bereich des Schwerpunktthemas zeigen. Ergänzt werden sie nach Möglichkeit durch einen Beitrag aus Patientensicht und durch die „Internationalen Studienergebnisse“, die auf aktuelle Studien aufmerksam machen, sowie durch den Artikel „Gelesen und kommentiert“, der die Durchführung und die Ergebnisse wichtiger Studien vorstellt und deren Schwächen und Stärken aufzeigt.

Die Schwerpunktthemen legt das Herausgeberteam im Rahmen der jährlichen Herausgebersitzung gemeinsam mit der Redaktion fest. Aufgabe der Herausgeber ist es, ausgewiesene Experten zum Schwerpunktthema zu suchen und als Autoren zu gewinnen. Die Herausgeber lesen die eingereichten Beiträge, prüfen die Texte auf Überschneidungen und Widersprüche mit anderen Artikeln und achten auf eine fristgerechte Einreichung der Manuskripte an den Verlag.

\section{Schatztruhe für therapeutisch relevante Informationen}

Vom ersten Heft bis zur aktuell vorliegenden Nummer bietet die Zeitschrift neuroreha einen wahren Schatz an therapeutisch relevanten Informationen. Exemplarisch kann dazu gleich das im November 2009 erschienene Heft Nr. 1 zum Thema „Repetition“ herangezogen werden.

In seinem Beitrag „Rehabilitation und Plastizität“ führte Christoph Globas unter anderem die zentralen Prinzipien neuronaler Reorganisation [3] auf:

- Synchrone Stimulation von Nervenzellverbänden führt zu einer engeren Vernetzung: „Fire together, wire together.“

- Vermehrte Stimulation/Bewegung führt zu einer Vergrößerung des entsprechenden kortikalen Repräsentationsareals.

- Nichtgebrauch führt zu einer Schrumpfung der kortikalen Repräsentation: „Use it or lose it.“

- Veränderungen werden nur induziert, wenn sie verhaltens-/aufgabenrelevant sind und so intensiv geübt werden, dass das Gehirn mit der Verarbeitung auch noch in den Schlaf hinein beschäftigt ist: „You have to dream it to achieve it."

Im Anschluss fokussierten Christian Haas und Klaus Blischke auf die „Bedeutung der Repetition für das motorische Lernen “ und übertrugen dafür die Lehren aus der Sportwissenschaft in die neurologische Rehabilitation. Dabei wiesen sie unter anderem auf folgende Punkte hin:
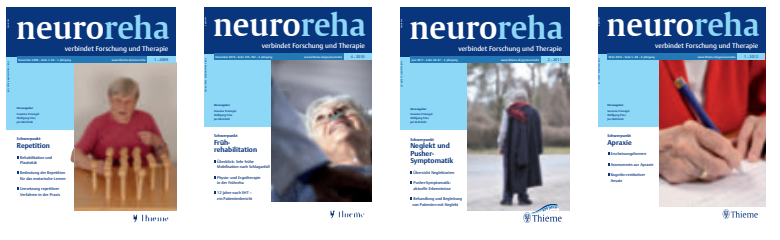

- Übung führt in der Regel zu einer energetischen Bewegungsoptimierung (=Verminderung der Kraftleistung), während initial die Freiheitsgrade durch eine Kokontraktion fixiert werden.

- Die Konsolidierung neu gelernter motorischer Fertigkeiten erfordert Zeit und Ruhe, das heißt, innerhalb von vier bis sechs Stunden nach Beendigung einer Lerneinheit sollten weder die neu gelernte noch ähnliche Fertigkeiten geübt werden.

- Automatisierung motorischer Fertigkeiten ist aufgabenabhängig und erfordert hohe bis sehr hohe Wiederholungszahlen.

Caroline Renner und Horst Hummelsheim komplettierten die Schwerpunktartikel aus einer klinischen Perspektive mit einem Beitrag über das „Komplexe aufgabenspezifische repetitive Training bei zentralmotorischen Störungen“. Sie forderten:

- Repetitives Training muss aktiv sein; bei hochgradigen Paresen wenn nötig unter Gewichtsabnahme.

- Das Training muss an der Leistungsgrenze des Patienten ansetzen, und der Schwierigkeitsgrad der Anforderung muss kontinuierlich gesteigert werden (= Shaping).

- Trainingsschwerpunkte sollten den Bedürfnissen des Patienten angepasst sein.

- Insbesondere bei Patienten mit neuropsychologischen Defiziten sollten aufgabenspezifisch Bewegungsabläufe geübt werden, die sich im Alltag des jeweiligen Patienten tatsächlich wiederfinden.

Nachdenklich stimmte uns schon damals der Beitrag einer Patientin zur stationären Reha, die berichtete: „Als Patientin hatte ich keinen Einfluss auf den Therapieplan, weder wurde ich einbezogen, noch hatte ich ein Mitspracherecht. Während der Therapien fragten mich einige Therapeuten nach meinen Zielen, und bei einigen von ihnen hatte ich das Gefühl, dass sie diese auch ernst nahmen. Doch insgesamt konnte ich mir nicht vorstellen, dass die Therapeuten - die oft wechselten - wussten, worauf es mir ankam und was mir wichtig war."

Wir fragen uns zehn Jahre später: „Werden diese zentralen Prinzipien der Rehabilitation mittlerweile flächendeckend umgesetzt? Sind es heute tatsächlich die Patientenziele, die das therapeutische Vorgehen bestimmen?“

\section{Stöbern im Archiv lohnt sich}

Beim Durchblättern der bisher erschienenen Hefte ist mir die große Fülle relevanter Inhalte aufgefallen, die wertvol-
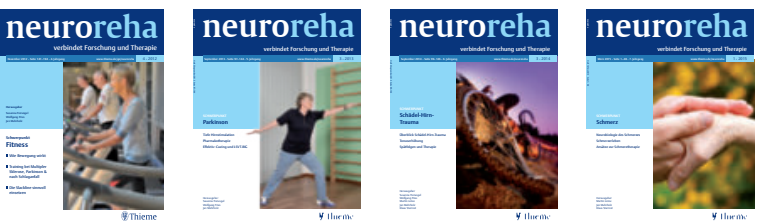
le Hinweise zum therapeutischen Vorgehen liefern. Jeder neuroreha-Abonnent findet sie im neuroreha-Archiv unter www.thieme-connect.de/products. Für den Zugang benötigt man lediglich die Abonnentennummer.

Auf einige Aspekte, die besonders viel zu diskutieren gaben, möchte ich bei der folgenden chronologischen Auflistung der Schwerpunktthemen kurz eingehen.

\section{Internationale Autoren ermöglichen einen Blick über den Tellerrand}

Schwerpunktthemen im Jahr 2010 waren: Gehen, Motivation, Tonuserhöhung, Frührehabilitation. Hier gab nicht nur das Heft zur Tonuserhöhung viel Anlass zu Diskussionen, sondern auch das Heft Frührehabilitation.

Während Anand D. Pandyan in seinem Beitrag eine neue Definition von Spastik vorschlug, die Spastik in meinen Augen nur unzureichend gegenüber den anderen Plussymptomen des „Upper-Motor-Neuron-Syndrom“ und auch von anderen Formen einer Tonuserhöhung abgrenzt, war er sich mit den Autorinnen Roberta B. Shepherd und Janet H. Carr einig, dass die Behandlung der Spastik - im Gegensatz zur Parese - nicht im Vordergrund der physiotherapeutischen Behandlung stehen sollte.

Im Heft über die Frührehabilitation stellt Julie Bernhardt die in Australien 2001 gestartete AVERT-Studie (= A Very Early Rehabilitation Trial) vor, die die „sehr frühe“ Rehabilitation mit einem Start der Mobilisation in den ersten 24 Stunden nach den Schlaganfallsymptomen definiert. Abgeklärt werden soll mit dieser Studie, an der weltweit viele Kliniken beteiligt sind, die Durchführbarkeit und Sicherheit einer frühen Mobilisation von Patienten nach Schlaganfall.

\section{Großes Themenspektrum vom konkreten Symptom über Leitlinien bis zur Versorgungsforschung}

Anfang 2011 folgten dann die Themen Willkürmotorik und Kognition sowie Neglekt und Pusher-Symptomatik. Darin stellten die Autoren die unterschiedlichen Neglektarten und Erklärungsmodelle vor sowie Skalen zur Erfassung einer Neglekt- und Pusher-Symptomatik und deren therapeutische Behandlungsansätze. Die Ergotherapeutin Friederike Kolster beschrieb darin auch die von ihr entwickelte „Handlungsorientierte Diagnostik und Therapie (HoDT) “ in der Neglekttherapie, die die subjektive Wahrnehmung des
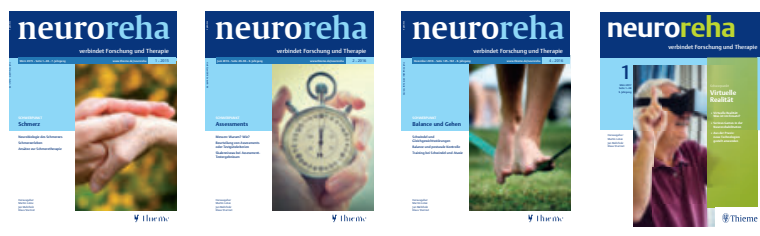

Betroffenen als gegeben akzeptiert und deshalb in einem ersten Schritt ipsiläsionales Handeln fördert, bevor Exploration und Handlungsfähigkeit kontraläsional gefordert werden. Weitere Schwerpunkte im Jahr 2011 waren das Thema Versorgungsforschung und Versorgungspraxis und schließlich ein umfassender Überblick zu Hand-Arm-Aktivitäten. Dieser bot nicht nur leitliniengestützte Therapieempfehlungen zum Schulter-Arm-Syndrom, sondern thematisierte auch die Evidenzlage unterschiedlicher Behandlungsmaßnahmen zur Verbesserung von Funktionen und Aktivitäten der oberen Extremität.

2012 folgten dann die Schwerpunkte Apraxie, Leitlinien, Wahrnehmung und Fitness. Während das Heft Apraxie umfassend über diese nach Schlaganfall der sprachdominanten Großhirnhemisphäre häufig auftretende kognitivmotorische Störung berichtete und dabei viele praktische, klinisch umsetzbare Informationen lieferte, war das auf den ersten Blick theoretische Heft zu Leitlinien mindestens genauso relevant für die Praxis. Das Heft stellte die Umsetzung und die internationale Anwendung von Leitlinien vor, erklärte die Wertung unterschiedlicher Leitlinien und beantwortete damit auch die Frage, welche Leitlinien bei gegebenenfalls unterschiedlichen Empfehlungen zu befolgen sind.

2013 standen die Themen CIP: Critical-IIlness-Polyneuropathie, Frontalhirn: Neuropsychologie und Verhalten, Parkinson sowie Schlucken auf der Agenda. Im Heft über das Schlucken gaben die von uns gefundenen Experten einen umfassenden Überblick zu evidenzbasierter Dysphagietherapie und Untersuchungstechniken des Schluckens. In ihrer Übersicht zu Therapiekonzepten in der Dysphagietherapie forderten Ulrike Starrost und Berit Schilling dazu auf, sich bei der Auswahl schlucktherapeutischer Maßnahmen weniger an therapeutischen Glaubensrichtungen und mehr an der verfügbaren Evidenz zu orientieren. Eine Forderung, die für alle therapeutischen Berufsgruppen gleichermaßen gilt und auch dafür sorgt, dass die neuroreha in therapeutischen Teams immer wieder Gesprächsthema ist.

\section{Neues Herausgeberteam bringt frische Ideen}

Ende 2013 stießen Martin Lotze und Klaus Starrost zu unserem Herausgeberteam. Mit ihnen waren wir noch breiter aufgestellt, um die Themen Multiple Sklerose, Gruppentherapien, Schädel-Hirn-Trauma und Demenz im Jahr 2014 mithilfe zahlreicher Experten aufzubereiten. Mit dem Heft zu Gruppentherapien griffen wir beispielsweise ein
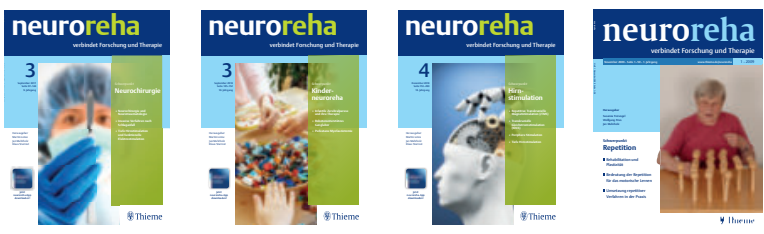
Thema auf, das bis dato in der auf Einzeltherapie fokussierten Behandlung wenig Beachtung fand. Eine differenzierte Betrachtung zeigt, dass die Vorteile der Gruppentherapie in der Bewegungsbeobachtung (Lernen am Modell der Mitpatienten), der Motivation und der sozialen Eingebundenheit liegen, dass aber die Einzeltherapie dann Vorteile hat, wenn spezifisch an der individuellen Leistungsgrenze geübt werden kann [5].

\section{Gleiche Themen neu betrachtet}

Ende 2014 haben Wolfgang Fries und ich das Herausgeberteam mit Beendigung unserer aktiven Berufstätigkeit verlassen. Dankbar sind wir dafür, dass wir die neuroreha mitgestalten konnten. Wir freuen uns über die Kreativität und Kompetenz der neuen Herausgeber.

2015 folgten dann die Themen Schmerz, Zukunftswerkstatt, Frühmobilisation und Training. Das Heft Schmerz informierte nicht nur über die Neurobiologie und das Schmerzerleben, sondern zeigte auch therapeutisch wertvolle Ansätze zur Schmerztherapie auf. Zwar war das Thema Frührehabilitation ja schon 2010 ein Schwerpunktheft der neuroreha, aber da mittlerweile die Ergebnisse der AVERT-Studie [1] vorlagen, griffen die Herausgeber das Thema erneut auf. Die Auswertung der Studie von Julie Bernhardt und ihrem Team zeigte, dass zwar eine „sehr frühe" Mobilisation und intensivere Therapie auch bei Patienten mit Lysetherapie durchführbar sind, die Patienten dieser Gruppe jedoch im Vergleich mit der Kontrollgruppe, die „usual care“ mit weniger intensiver Therapie erhielt, nach drei Monaten eine schlechtere Erholung zeigten. Festzuhalten ist, dass die Zeit bis zur Mobilisation der „sehr frühen " Gruppe im Schnitt 18,5 Stunden betrug, während die Patienten der Kontrollgruppe nach durchschnittlich 22,4 Stunden mobilisiert wurden. Empfehlungen zu einer frühen Mobilisation sind deshalb mittlerweile Bestandteil vieler Leitlinien zur Schlaganfallversorgung geworden. Ein Beitrag zu Kontraindikationen für eine frühe Mobilisation und zu Folgen von Bettruhe und Immobilisation ergänzten dieses Thema und vermittelten dem Leser damit einen umfassenden und aktuellen Überblick zum therapeutischen Vorgehen bei der Mobilisation von Patienten nach Schlaganfall.

\section{Von Früh- bis Spätreha und von Lähmung bis zum Gehen}

Nur konsequent war, dass dann 2016 auch die Spätreha mit der Wiedereingliederung in das Arbeitsleben einen eigenen Schwerpunkt erhielt. Mit dem Heft über Assess-
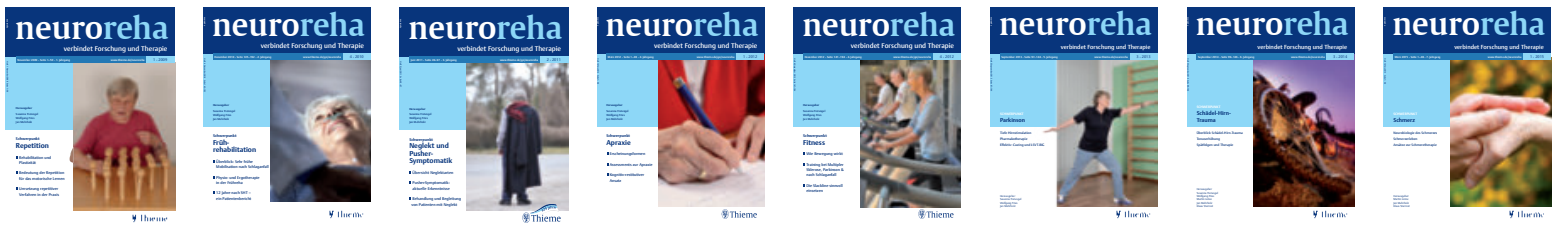

ments ging die neuroreha danach auf die Bedeutung, Durchführung und Interpretation von Messverfahren ein und stellte eine Übersicht valider Testverfahren der neurologischen Rehabilitation vor. Ein konkretes Krankheitsbild folgte in Heft 3/2016 zum Schwerpunkt „Neuroreha nach Querschnittlähmung“, während in Heft 4 die auf viele Patienten zutreffenden Zielkriterien „Balance und Gehen“ aufgegriffen wurden. Dort konnten sich die Leser sowohl über Schwindel und Gleichgewichtsstörungen als auch über Gehen und posturale Kontrolle nach Schlaganfall informieren. Während Klaus Jahn in seinem Beitrag Ursachen und Formen des Schwindels aufzeigte, informierte Matthias Wiemer praxisorientiert über die Durchführung eines Trainings bei Schwindel. Simone Thomas und Jan Mehrholz betonten in ihrem Beitrag, dass bei Patienten nach Schlaganfall Gehen unabhängig von Sitz- und Stehbalance mittels Laufbandtraining geübt werden kann: „Stepping before standing“ [4]. Zudem wiesen sie anhand einer Studie von Choi darauf hin, dass sich bei diesen Patienten die posturale Kontrolle dann verbessert, wenn während des Gehens spezifische posturale Anforderungen gestellt werden [2].

\section{Immer im Blick: der technische Fortschritt}

2017 stand im Zeichen moderner technischer Hilfsmittel, die für die Neurorehabilitation künftig zunehmend relevant werden. Im Schwerpunktheft Virtuelle Realität erhielten die Leser neben einem Übersichtsartikel zum Einsatz virtueller Realität in der Neurorehabilitation auch Erklärungen zu den Unterschieden von Virtual Reality (VR) und Augmented Reality (AR). So konnte man erfahren, dass bei VR die reale Welt komplett ausgeschlossen wird, während AR eine computergestützte Erweiterung der menschlichen Wahrtion, bedeutet. Betont wird, dass es für rehabilitative Anwendungen wichtig ist, dass sich das Schwierigkeitsniveau an das Niveau des Spielenden anpasst und herausfordernd, aber nicht frustrierend ist. In einer Metaanalyse von zehn Studien zur virtuellen Realität zeigte sich für die obere Extremität zwar (noch?) keine Überlegenheit im Vergleich zur Standardtherapie, überlegen war VR aber bei der Verbesserung von Balanceleistungen. Ende des Jahres folgte dann ein Heft zur Robotik, in dem verschiedene assistierende Robotersysteme vorgestellt wurden. Weitere Schwerpunkte waren 2017 und 2018 Aphasie, Neurochirurgie, Musiktherapie, Psychologische Begleiterkrankungen, Kinderneuroreha und schließlich die Übersicht über die heutigen Möglichkeiten moderner Hirnstimulationsverfahren. nehmung, z. B. durch Einblenden visueller Zusatzinforma- 
Mit dieser subjektiven Auswahl einiger Details aus den letzten Jahren möchte ich dazu anregen, die Hefte der letzten Jahre ruhig noch mal anzusehen und auch bisher nicht bekannte Ausgaben kennenzulernen. Das kann seit Ende 2016 übrigens auch in der praktischen Form der „neuroreha-App“ passieren, in der für Abonnenten alle Beiträge der Hefte und die jeweiligen Literaturverzeichnisse jederzeit verfügbar sind.

\section{Wie kann die neuroreha Forschung und Therapie noch besser verbinden?}

Als Rückmeldung von Kollegen und Kolleginnen haben wir in den letzten zehn Jahren häufig gehört: „Die neuroreha verlangt ihren Lesern viel ab und setzt neues Denken voraus." Das stimmt sicher, ist aber doch gerade das, was notwendig ist, um den Wissensstand und die therapeutischen Vorgehensweisen in Deutschland zu verändern und dem internationalen Niveau anzugleichen.

Die Fragen, die sich Verlag und Herausgeber immer wieder stellen, sind:

- Was wäre für die Leserinnen und Leser hilfreich, um den erwünschten Wissenstransfer in die Praxis zu unterstützen?

- Was braucht es, um eine ideologiefreie Diskussion über therapeutische Ansätze und die Überprüfung von deren Wirksamkeit zur Evaluation des optimalen therapeutischen Vorgehens zu ermöglichen?

- Wie können die dafür notwendigen Voraussetzungen einer gemeinsamen Sprache/Terminologie, aktueller medizinischer und physiologischer Erkenntnisse und grundlegender wissenschaftlicher Studien zur Überprüfung der Wirksamkeit von Therapieansätzen vermittelt werden?

In der Vermittlung von Wissen hat die neuroreha in der Vergangenheit zweifellos einen sehr wichtigen Beitrag geleistet. Wünschenswert wäre es, wenn aus den Reihen der Therapeuten Vorschläge zu den oben skizzierten Fragen kommen würden, um die Zeitschrift in Zukunft noch näher an die Bedürfnisse und Fragen der praktisch tätigen Therapeuten anzupassen. Denn dann könnte die Zeitschrift ihrem Anspruch „verbindet Forschung und Therapie“, den sie in ihrem Untertitel formuliert, auch im therapeutischen Alltag noch besser gerecht werden.

Unabhängig davon ist natürlich ein wichtiges zukünftiges Ziel, dass das in der neuroreha thematisierte evidenzbasierte therapeutische Vorgehen Eingang in die Gesundheits-
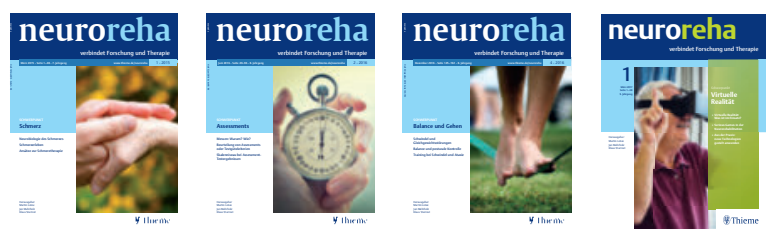

politik in Deutschland findet und dieses Grundlage für die Diskussion des gemeinsamen Bundesausschusses zur Vergütung der Heilmittelerbringer wird.

\section{Autorinnen/Autoren}

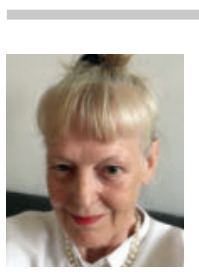

\section{Susanna Freivogel}

Ist Physiotherapeutin und hat über 20 Jahre die Abteilung Physiotherapie im HegauJugendwerk in Gailingen geleitet. Seit 2009 übt sie eine selbstständige Beratungstätigkeit auf dem Gebiet der Neurorehabilitation aus und unterrichtet in einem Bachelor- und Masterstudiengang für Neurorehabilitation.

\section{Korrespondenzadresse}

\author{
Susanna Freivogel \\ Physiotherapie, Fortbildung und Beratung \\ Freyastrasse 16 \\ $\mathrm{CH}-8212$ Neuhausen \\ E-Mail: s.freivogel@gmx.net
}

Literatur

[1] AVERT Trial Collaboration group. Efficacy and safety of very early mobilisation within $24 \mathrm{~h}$ of stroke onset (AVERT): A randomised controlled trial. Lancet 2015; 386(9988): 46-55

[2] Choi W, Lee G, Lee S. Effect of cognitive-motor dual task using auditory cue on balance of survivors with chronic stroke: A pilot study. Clin Rehabil 2014; 8: 763-770

[3] Elbert T, Rockstroh B. Reorganization of human cerebral cortex: The range of changes following use and injury. Neuroscientist 2004; 10(2): 129-141

[4] Horn S, Dejong G, Smout R et al. Stroke rehabilitation patients, practice and outcome: Is earlier and more aggressive therapy better? Arch Phys Med Rehabil 2005; 12(51): 101-114

[5] Miklitsch C, Krewer C, Freivogel S, Steube D. Effects of a predefined mini-trampoline training programme on balance, mobility and activities of daily living after stroke: A randomized controlled pilot study. Clin Rehabil 2013; 27(10): 939-947

Bibliografie

DOI https://doi.org/10.1055/a-0825-8133

neuroreha 2019; 11: 9-13

(c) Georg Thieme Verlag KG Stuttgart · New York ISSN 1611-6496
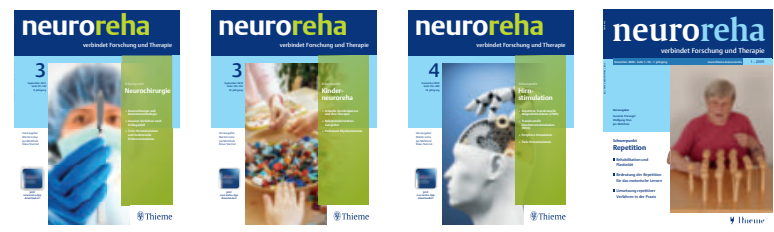